\title{
Follow My Finger Navigation
}

\author{
Rami Ajaj, Frédéric Vernier, and Christian Jacquemin \\ LIMSI-CNRS and Université Paris-Sud 11 \\ B.P. 133 / 91403 Orsay Cedex \\ France \\ \{rami.ajaj, frederic.vernier, christian.jacquemin\}@limsi.fr
}

\begin{abstract}
This paper presents a novel interaction technique called Follow my Finger $(\mathrm{FmF})$ for navigation in $3 \mathrm{D}$ virtual environments using a $2 \mathrm{D}$ interactive view on a table-top device. FmF consists in moving a camera icon that represents the 2D subjective position and orientation of a viewpoint in the 3D world. Planar, tactile, and direct manipulation of the camera icon facilitates navigation in the 3D environment. From the user's perspective the camera icon follows her/his finger trajectory to interactively modify the horizontal location, inclination, and orientation of the 3D point of view.
\end{abstract}

Keywords: Table-top, navigation, interaction technique, 3D environments.

\section{Introduction}

Navigation techniques in 3D virtual environments have been developed for decades but each present advantages and drawbacks. A mouse and/or a keyboard are often used for navigation tasks in desktop 3D environments such as video games or computer-aided design applications. The use of these input devices seems less intuitive but faster and more accurate than direct touch of table-top devices [1]. This article studies the combination of 3D and 2D visualization and a corresponding interaction technique that facilitates intuitive navigation in virtual worlds through direct touch interactions (see Fig. 1).

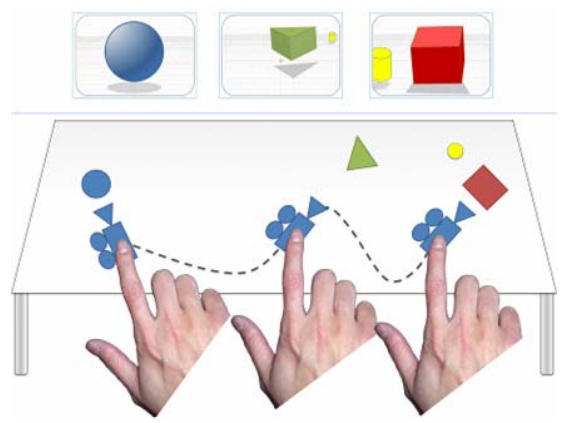

Fig. 1. FmF navigation. Bottom: The user's finger control of the camera icon. Top: The corresponding $3 \mathrm{D}$ view at 3 camera key positions and orientations.

T. Gross et al. (Eds.): INTERACT 2009, Part II, LNCS 5727, pp. 228 231, 2009.

(C) IFIP International Federation for Information Processing 2009 


\section{Setup Description}

The new interaction technique introduced in this article has been developed in a more complete framework for the combination of 2D and 3D interaction named Management Cabin (MC). MC is an architecture for multimodal interaction in 3D virtual worlds. It is made of a 2D interactive horizontal view of a virtual scene projected on a table-top device and a 3D view of the same scene presented vertically close to the table-top device in front of the user (see Fig. 2 left). A camera icon is displayed in the $2 \mathrm{D}$ table-top view and represents the point of view (POV) position and orientation in the 3D view. The figure 2 illustrates a user interacting through the table-top device and looking at the $3 \mathrm{D}$ view presented in front of her/him.

The MC software architecture is based on three components (see Fig. 2 right) that manage complementarity, consistency, and concurrency between the two interfaces (2D and $3 \mathrm{D}$ view and interaction). The central component controls the communication between the $2 \mathrm{D}$ and the $3 \mathrm{D}$ components.
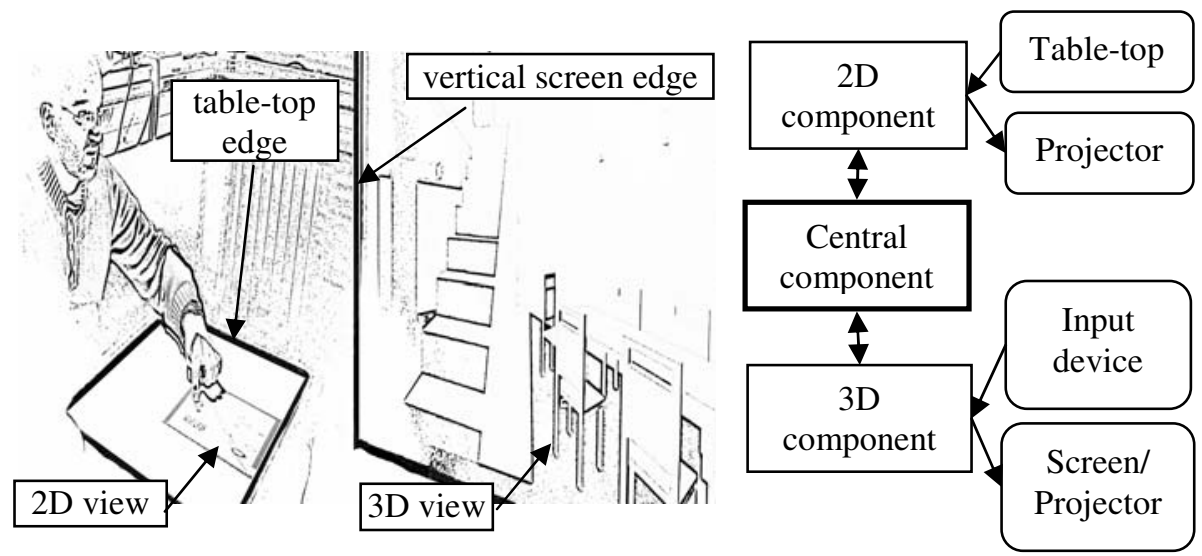

Fig. 2. Left: A user interacting with the $2 \mathrm{D}$ table-top view. Middle: The vertical screen in front of the user with the 3D view. Right: The software components and their connections to the input and output devices in MC.

\section{Follow My Finger Navigation Technique}

FmF Interaction Technique Description. At the 2D only level, the FmF navigation technique is similar to the Rotate' $N$ Translate (RNT) interaction technique [2]. In RNT, window or 2D object orientation is automatically linked to the trajectory of interaction. In 3D, orientation can be automatically linked to the relative position of the camera and a virtual object like in HooverCam [3]. In FmF and RNT, orientation is deduced from the trajectory of the drag and drop. RNT is used directly for window manipulation while FmF uses a 2D camera icon linked to a 3D point of view for 3D navigation (like the HooverCam). FmF allows a user to select a camera icon with her/his finger and, while dragging the icon on the table, the camera orientation follows the trajectory. This technique controls both $2 \mathrm{D}$ rotation and $2 \mathrm{D}$ translation 
transformations of the 3D viewpoint. However translations and rotations can-not be performed separately.

Planar and direct manipulations of the camera icon allow easy and intuitive navigation in a 3D virtual environment. Moreover, simultaneous rotation and translation offer fast navigation task accomplishment when compared with interaction techniques that alternate translation and rotation tasks. A convincing example to illustrate these advantages is to consider a user navigating in a $3 \mathrm{D}$ scene that contains three objects of interest (see Fig. 1) in which she/he wants to visualize different key POVs in the 3D view.

The effect of the FmF interaction technique on the 3D navigation is a fluid and coherent navigation. Moreover, it allows a 2D direct manipulation of the 3D POV. RNT applied to 3D navigation does not cover the full range of possible degrees of freedom (DoF): vertical translation, pitch and roll are not covered. Among the three missing DoFs, the pitch is the most important for walk through navigation. Based on our observations, pitch and orientation seem related to trajectory features: while running fast we tend to look downward and looking upward is related to back movements. We have transposed these observations to FmF to control inclination of the viewpoint through fast or slow motions along the trajectory (forward=looking down and backward=looking up). To allow backward motions, we disable orientation linking to the trajectory for very sharp rotation angles $\left( \pm 60^{\circ}\right.$ around the opposite direction to trajectory). To better match walk through navigation, we apply a threshold to the pitch angle to fit human neck capabilities $\left( \pm 60^{\circ}\right.$ around horizontal). We manually adapted the pitch angle to the navigation speed according to table size and resolution. The pitch angle is modified linearly with respect to speed when navigating in the trajectory direction. We choose not to apply this linearity between pitch angle and speed to backward navigation to avoid users' dizziness. Therefore, pitch angle goes from $0^{\circ}$ to the maximum threshold and stays fix during backward navigation. At last, two possibilities exist at finger release: the last pitch angle is preserved or it is put back to the relaxed angle $\left(0^{\circ}\right)$ with or without animation.

Multiple Cameras. Several camera icons can be displayed in the 2D view. Fingerbased manipulation allows fast and easy POV change. In the case of one vertical display, only one camera, which represents the POV of the current 3D view, can be active. The POV change is made by choosing a button on the selected camera that will activate it. The button use allows an "offline" POV positioning and orientation (without inclination) by manipulating an inactive camera icon. The 3D POV is automatically moved to the new position that corresponds to the new current camera position and orientation in the $3 \mathrm{D}$ scene.

Multiple camera representations facilitate fast and easy POV modifications in scenes with multiple canonical views. For example a given POV might be important for a user who also wishes to explore other POVs in the virtual scene (e.g. in figure 1 the user wants to easily return to the POV in front of the sphere); In this case the user adds a new camera icon and navigates using this camera and return to the selected view through just one click. The use of multiple camera icons helps to overcome the lack of lateral translation in FmF. 
Translated and Hybrid Interaction Techniques. In addition to FmF, we have implemented two other interaction techniques: the translated and the hybrid interaction techniques. The translated navigation technique allows the translation of the 3D POV horizontally through direct manipulation of the camera icon on the $2 \mathrm{D}$ view with user's finger. For rotation tasks, a widget is presented to the user next to the camera icon. The direct selection and manipulation of this widget leads to the 3D POV rotation. In comparison with the FmF technique, the translated technique allows separate and not simultaneous rotation and translation of the 3D POV. Moreover, the inclination is not managed in the translated technique.

The hybrid interaction technique combines both, translated and FmF, interaction techniques and offers the user the possibility to choose easily between both. Inspired by $[2,4]$, we divide the selection area of the camera icon into two parts. The front selection area of the camera icon corresponds to the $\mathrm{FmF}$ interaction technique whereas the back selection area corresponds to the translated navigation mode. This interaction technique allows simultaneous as well as separate 3D POV rotation and translation. The drawback is the toggle between two interaction techniques that could disorient users.

\section{Discussion and Conclusion}

We have implemented FmF in $\mathrm{MC}$ and used it to navigate in a small, two floors house model. The resulting interaction technique matches our expectations and the four DoFs are seamlessly integrated. However the parameters must be adjusted with end users and a user experiment must be conducted to study the assimilation of the four DoFs. FmF should also be compared to other navigation techniques that allow four DoFs. At last, we foresee how our extension to RNT [2] can also be applied to TNT [4] and provide a new family of very intuitive $3 \mathrm{D}$ navigation techniques by using the physical finger orientation.

\section{References}

1. Shen, C., Ryall, K., Forlines, C., Esenther, A., Vernier, F.D., Everitt, K., Wu, M., Wigdor, D., Morris, M.R., Hancock, M., Tse, E.: Informing the Design of Direct-Touch Tabletops. IEEE Comput. Graph. Appl. 26(5), 36-46 (2006)

2. Kruger, R., Carpendale, S., Scott, S.D., Tang, A.: Fluid integration of rotation and translation. In: Proc. of the SIGCHI Conference on Human Factors in Computing Systems CHI 2005, April 02 - 07, 2005, pp. 601-610. ACM, New York (2005)

3. Khan, A., Komalo, B., Stam, J., Fitzmaurice, G., Kurtenbach, G.: HoverCam: interactive 3D navigation for proximal object inspection. In: Proc. of the 2005 Symposium on interactive 3D Graphics and Games, I3D 2005, Washington, District of Columbia, April 03 - 06, 2005, pp. 73-80. ACM, New York (2005)

4. Liu, J., Pinelle, D., Sallam, S., Subramanian, S., Gutwin, C.: TNT: improved rotation and translation on digital tables. In: Proc. of Graphics interface 2006, Quebec, Canada, June 07 09, 2006, vol. 137, pp. 25-32. Canadian Information Processing Society, Toronto (2006) 